\title{
Removal of AMPA Receptors (AMPARs) from Synapses Is Preceded by Transient Endocytosis of Extrasynaptic AMPARs
}

\author{
Michael C. Ashby, ${ }^{1}$ Sarah A. De La Rue, ${ }^{1}$ G. Scott Ralph, ${ }^{1,2}$ James Uney, ${ }^{2}$ Graham L. Collingridge, ${ }^{1}$ and Jeremy M. Henley ${ }^{1}$ \\ ${ }^{1}$ Medical Research Council (MRC) Centre for Synaptic Plasticity, Department of Anatomy, School of Medical Sciences, University of Bristol, Bristol BS8 \\ 1TD, United Kingdom, ${ }^{2}$ MRC Centre for Synaptic Plasticity, Division of Medicine, University of Bristol, Bristol BS2 8HW, United Kingdom
}

\begin{abstract}
AMPA receptors (AMPARs) are dynamically regulated at synapses, but the time course and location of their exocytosis and endocytosis are not known. Therefore, we have used ecliptic pHluorin-tagged glutamate receptor 2 to visualize changes in AMPAR surface expression in real time. We show that synaptic and extrasynaptic AMPARs respond very differently to NMDA receptor activation; there is a rapid internalization of extrasynaptic AMPARs that precedes the delayed removal of synaptic AMPARs.
\end{abstract}

Key words: AMPARs; trafficking; imaging; cell surface; pHluorin; hippocampus

\section{Introduction}

AMPA receptor (AMPAR) trafficking to and from the postsynaptic membrane is involved in establishing the activity-induced changes in synaptic strength associated with learning and memory formation (Malinow and Malenka, 2002). The number of synaptic AMPARs is determined by the balance between insertion and removal of receptors at the postsynaptic site. There is compelling evidence that these processes are highly dynamic and tightly regulated, likely involving exocytosis, endocytosis, and lateral diffusion in the plasma membrane (Morales and Goda, 1999; Malinow et al., 2000; Carroll et al., 2001; Passafaro et al., 2001; Sheng and Lee, 2001; Borgdorff and Choquet, 2002). However, the precise localization and time course of these processes have not been resolved, in large part because of limitations in the experimental approaches that have been available.

Recombinant, tagged receptors have been used extensively to investigate the behavior of AMPAR subunits in neurons (Lissin et al., 1998; Passafaro et al., 2001; Shi et al., 2001). In particular, tagging with green fluorescent protein (GFP) and confocal microscopy have provided major insight into the dynamics of receptor trafficking in living cells. A significant limitation with conventional GFP-tagged receptors is that it is not possible to directly distinguish surface from intracellular fluorescence. One approach to overcome this limitation has used a thrombin cleavage assay combined with antibody labeling to measure the rate and

Received March 22, 2004; revised April 18, 2004; accepted April 23, 2004.

This work was supported by the Wellcome Trust, the Medical Research Council, and European Union Grant QLRT-2000-02089. We are grateful to Professor George Banting and Dr. Andrew Doherty for useful advice. We also thank Dr. J. E. Rothman for the original pHluorin CDNA.

Correspondence should be addressed to J. M. Henley, Department of Anatomy, School of Medical Sciences, University of Bristol, Bristol BS8 1TD, UK. E-mail: j.m.henley@bristol.ac.uk.

G. S. Ralph's present address: Oxford BioMedica (UK) Limited, Medawar Centre, Robert Robinson Avenue, The Oxford Science Park, Oxford 0X4 4GA, UK.

DOI:10.1523/JNEUROSCI.1042-04.2004

Copyright $\odot 2004$ Society for Neuroscience $\quad$ 0270-6474/04/245172-05\$15.00/0 location of surface expression of AMPAR subunits (Passafaro et al., 2001). However, this technique has very limited temporal resolution.

Here we report an alternative strategy that uses $\mathrm{pH}$-sensitive GFP (ecliptic pHluorin) to visualize surface-expressed glutamate receptor 2 (GluR2) receptors in real time at individual synapses. Characterization of the properties of pHluorin-GluR2 shows that the recombinant protein behaves like endogenous GluR2. We have used this tool for high-resolution imaging studies and demonstrate that the surface-expressed synaptic and extrasynaptic AMPARs are subjected to distinct patterns of NMDA receptor (NMDAR)-dependent internalization. We show that synaptic and extrasynaptic AMPARs respond very differently to NMDAR activation; there is a rapid internalization of extrasynaptic AMPARs that precedes the delayed removal of synaptic AMPARs.

\section{Materials and Methods}

Construction of pHluorin-GluR2. Ecliptic pHluorin was engineered into rat GluR2 by overlapping PCR. The construct was assembled in pcDNA1/Amp vector (Invitrogen, San Diego, CA). The pHluorinGluR2 adenovirus was constructed using previously described methods (Harding et al., 1997).

Hippocampal neuronal culture. Primary hippocampal cultures were prepared from embryonic day 18 rat pups using standard protocols (Banker and Goslin, 1998). Cells were infected with adenovirus after $15-28 \mathrm{~d}$ in culture and used $48-72 \mathrm{hr}$ later. For experiments, neurons were transferred to HEPES-buffered saline (HBS) containing (in mM): $140 \mathrm{NaCl}, 5 \mathrm{KCl}, 25 \mathrm{HEPES}, 10$ glucose, $1.5 \mathrm{MgCl}_{2}$, and $2 \mathrm{CaCl}_{2}$, pH 7.4.

Live-cell confocal imaging. pHluorin-GluR2 fluorescence was excited by $488 \mathrm{~nm}$ laser light, and emission from a single confocal plane (depth between 2 and $4 \mu \mathrm{m}$ ) was detected through either 505 long-pass or 505530 bandpass filters (LSM510 META confocal system; Zeiss, Oberkochen, Germany). In initial optimization experiments, the imaging parameters were established to ensure minimal photobleaching of fluorescence ( $\sim 5 \%$ of initial fluorescence levels). Furthermore, in unstimulated neurons, pHluorin-GluR2 fluorescence was stable during the 
imaging experiments ( $n=3$; time, $>27 \mathrm{~min}$; data not shown). All imaging was done on the heated stage (set at $37^{\circ} \mathrm{C}$ ) using a $63 \times$ waterimmersion objective (numerical aperture, 1.2) of an inverted microscope (Zeiss Axiovert 200M), and neurons were continually perfused at $\sim 2$ $\mathrm{ml} / \mathrm{min}$ with solutions also heated to $37^{\circ} \mathrm{C}$. During analysis, a slight rise in fluorescence just before application of NMDA was observed. We attribute this to a small perfusion system-associated artifact. Bathing solutions were adjusted to appropriate $\mathrm{pH}$ using $\mathrm{NaOH}$ or $\mathrm{HCl}$. Neurons were stained with $5 \mu \mathrm{M}$ FM4-64 (FM) using the following protocol: HBS plus FM for $60 \mathrm{sec}$, KHBS (HBS containing $90 \mathrm{~mm} \mathrm{KCl}$ to depolarize cells) plus FM for $60 \mathrm{sec}$, HBS plus FM for $60 \mathrm{sec}$, and multiple HBS washes for $5 \mathrm{~min}$. FM4-64 fluorescence was excited using $488 \mathrm{~nm}$ light, and emitted light was collected through a long-pass $560 \mathrm{~nm}$ filter. When $\mathrm{NH}_{4} \mathrm{Cl}$ was used to collapse $\mathrm{pH}$ gradients, $50 \mathrm{mM} \mathrm{NH}_{4} \mathrm{Cl}$ was added to HBS in place of equimolar $\mathrm{NaCl}$. Temporal analysis of fluorescence is shown as changes compared with resting (initial) levels $\left(\Delta F / F_{\mathrm{o}}\right)$. The surface fraction of fluorescence was calculated using the peak $\Delta F / F_{\mathrm{o}}$ during application of $\mathrm{NH}_{4} \mathrm{Cl}$-containing $\mathrm{HBS}$, pH 7.4 (Sankaranarayanan et al.,2000). For thesecalculations, we estimated that intracellular pHluorinGluR2 resides in compartments with an average $\mathrm{pH}$ of 6.0 . This was based on the fact that, in dendritic regions, there was no decrease in fluorescence when low $\mathrm{pH}$ was applied to the interior of the cell compared with when it was applied only to the outside. In contrast to dendritic regions, in the cell body some intracellular fluorescence could often be observed and quenched (see Fig. $1 b$ ). This is likely attributable to the large amount of intracellular compartments and the relatively neutral $\mathrm{pH}$ (i.e., more endoplasmic reticulum) in these regions of the cell. For this reason, our analysis was restricted to dendritic areas. Very occasionally, small intracellular areas of bright fluorescence (seen during acid wash) were also identified in dendrites; regions of analysis were positioned to avoid these areas. Regions for analysis were selected manually and positioned to account for any small movements of the cell during the experiment. If substantial movement in the $x-y$ plane or in focus occurred, the experiment was discarded.

Immunocytochemistry. Neurons plated on coverslips were fixed in 4\% paraformaldehyde in HBS for $10 \mathrm{~min}$. For intracellular localization, cells were permeabilized with $0.1 \%$ Triton X-100 for $10 \mathrm{~min}$. All of the following antibody incubations were performed in 3\% BSA-containing HBS for $40 \mathrm{~min}$ at room temperature: $\mathrm{pHluorin}-\mathrm{GluR} 2$ was detected using rabbit polyclonal anti-GFP antibody (10 $\mu \mathrm{g} / \mathrm{ml}$; Chemicon, Temecula, CA), surface GluR1 was stained with an N-terminal-directed rabbit polyclonal anti-GluR1 antibody (10 $\mu \mathrm{g} / \mathrm{ml}$; Oncogene Research Products, San Diego, CA), and synapses were labeled with rabbit polyclonal antisynaptophysin antibody ( $5 \mu \mathrm{g} / \mathrm{ml}$; Calbiochem, La Jolla, CA). After washing, appropriately labeled secondary antibodies were applied for 40 min at room temperature. The following fluorescent tags were used: Alexa Fluor 594 and Alexa Fluor $564(2 \mu \mathrm{g} / \mathrm{ml}$; Molecular Probes, Eugene, OR), FITC ( $2 \mu \mathrm{g} / \mathrm{ml}$; Sigma, St. Louis, MO), and Cy5.5 (2 $\mu \mathrm{g} / \mathrm{ml}$; Abcam). Immunofluorescent staining was imaged using a $63 \times$ oil immersion lens on either a Leica (Nussloch, Germany) SP2 or a Zeiss LSM510 confocal microscope.

\section{Results}

We have used $\mathrm{pH}$-sensitive GFP (ecliptic pHluorin) to visualize surface-expressed GluR2 receptors in real time at individual synapses. Ecliptic pHluorin is essentially nonfluorescent at $\mathrm{pH}$ values of $<6.0$, and its brightness increases with $\mathrm{pH}$ values of up to $\sim 8.5$ (Miesenbock et al., 1998). Plasma membrane-targeted proteins are delivered to the surface via the secretory pathway in vesicular compartments that have an acidic lumen. Likewise, endocytosed plasma membrane forms vesicles that are rapidly acidified (Demaurex, 2002). We inserted ecliptic pHluorin in the extracellular $\mathrm{N}$-terminal domain of AMPAR subunits that orientate to the lumen of vesicles (Fig. 1a). Therefore, inside the cell, pHluorinGluR2 exhibits very low fluorescence, because it is in an acidic environment. Surface-expressed pHluorin-GluR2, in contrast, should become brightly fluorescent after exposure to an extracellular $\mathrm{pH}$ of $\sim 7.4$.

\section{pHluorin-GluR2 allows specific visualization of AMPARs in the plasma membrane of living neurons}

Experiments were performed at $37^{\circ} \mathrm{C}$ using cultured dissociated hippocampal neurons that expressed pHluorin-GluR2 after gene delivery via adenovirus. Confocal fluorescence microscopy revealed that the majority of fluorescence was localized in bright areas at the edges of dendrites, with much less fluorescence in the center of dendritic shafts (Fig. 1bi). Immunostaining with antiGFP antibody in living nonpermeabilized cells showed a similar surface distribution, indicating that pHluorin-GluR2 fluorescence accurately reports AMPARs at the cell surface (Fig. 1c). Almost all bright and punctate dendritic fluorescence was reversibly eliminated at low extracellular $\mathrm{pH}(6.0)$ because of the eclipse of pHluorin (Fig. 1bii). This brief acidic wash does not affect intracellular $\mathrm{pH}$, as measured by BCECF-AM (2',7'-bis-(2carboxyethyl)-5-(and 6)-carboxyfluorescein, acetoxymethyl ester; $n=3$; data not shown), confirming that pHluorinGluR2 fluorescence reports the specific localization of GluR2 at the dendritic surface in neurons.

\section{AMPARs containing pHluorin-GluR2 are delivered to functional synapses}

To establish the fraction of surface-expressed pHluorin-GluR2, we incubated neurons with $\mathrm{NH}_{4} \mathrm{Cl}$ to reversibly collapse intracellular $\mathrm{pH}$ gradients and measured fluorescence changes in dendrites (Fig. 1d). When the extracellular $\mathrm{pH}$ was low, addition of $\mathrm{NH}_{4} \mathrm{Cl}$ did not cause any additional reduction in fluorescence in dendritic regions. These data confirm that intracellular pHluorin-GluR2 is already eclipsed, and that any remaining fluorescence is attributable to autofluorescence. In contrast, $\mathrm{NH}_{4} \mathrm{Cl}$ in normal extracellular $\mathrm{pH}$ (7.4) caused a rapid rise in fluorescence as the intracellular pHluorin-GluR2 was revealed. From these values, we calculated that $\sim 45 \%$ of pHluorin-GluR2 is surface expressed under resting conditions (Sankaranarayanan et al., 2000). To test whether pHluorin-GluR2 accumulates at functional synapses, live pHluorin-GluR2-expressing neurons were stained with the presynaptic marker FM4-64 (Fig. 1e). Approximately three-quarters of pHluorin-GluR2 puncta were associated with active presynaptic terminals. In addition, immunolabeling showed that the vast majority of pHluorin-GluR2 puncta colocalize with endogenous GluR1-containing AMPARs and with the synaptic marker synaptophysin (Fig. 1f). This indicates that pHluorin-GluR2 is targeted to synapses that contain endogenous AMPARs.

\section{Synaptic and extrasynaptic AMPARs are subject to distinct patterns of NMDAR-dependent internalization}

Brief bath application of NMDA to hippocampal slices causes a chemically induced form of long-term depression (Chem-LTD) (Lee et al., 1998) and, in dispersed hippocampal cultures, exposure to NMDA evokes internalization of synaptic GluR1- and GluR2-containing AMPARs (Beattie et al., 2000; Ehlers, 2000; Lee et al., 2002). There is some indirect evidence that clathrindependent endocytosis of AMPARs does not occur at the postsynaptic density (PSD) (Blanpied et al., 2002), but the sites and kinetics of AMPAR internalization have not been defined. The good signal-to-noise ratio of pHluorin-GluR2 allowed us to clearly visualize both bright punctate synaptic fluorescence and lower levels of diffuse extrasynaptic fluorescence. We have therefore been able, for the first time, to directly compare internaliza- 

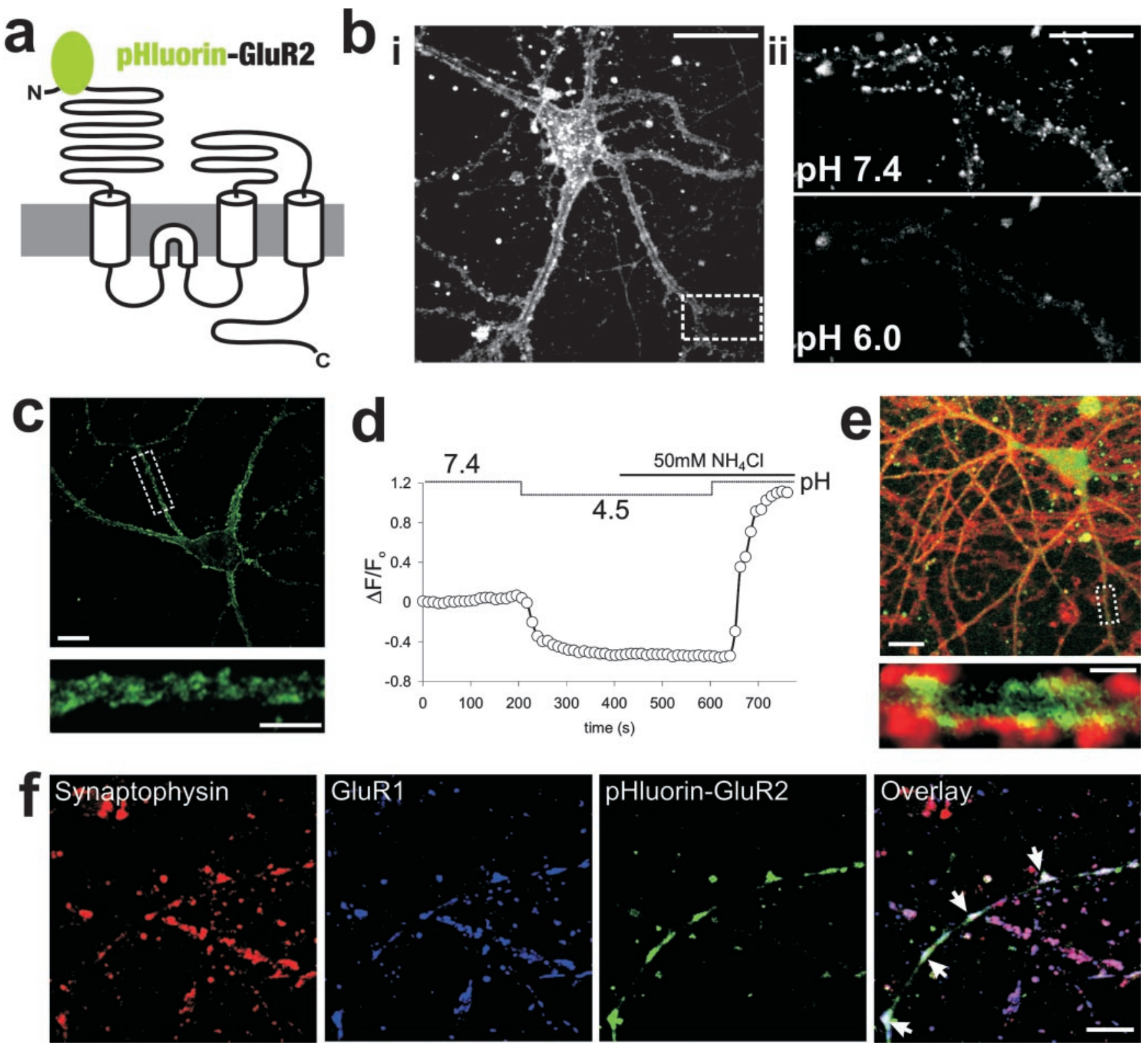

Figure 1. pHluorin-GluR2 reports the dynamic surface expression of AMPARs. $a$, Schematic of pHluorin-GluR2. b, Confocal image of typical pHluorin-GluR2 fluorescence distribution in a live, cultured hippocampal neuron. $i$, Fluorescence localizes predominantly to the edges of dendritic shafts. Scale bar, $20 \mu \mathrm{m}$. ii, The punctate, dendritic pHluorin-GluR2 fluorescence (from the dashed box in bi) seen at normal pH (7.4) is removed by washing cells with extracellular solution with reduced pH (6.0). Scale bar, $10 \mu \mathrm{m}$. c, Fluorescence distribution of anti-GFP surface (unpermeabilized) immunostaining from a pHluorin-GluR2-expressing neuron. Scale bar, $10 \mu \mathrm{m}$. The dendritic region indicated by the dashed white box in the top image is magnified in the bottom image. Scale bar, $5 \mu \mathrm{m} . d$, Averaged pHluorin-GluR2 fluorescence changes from dendritic regions of a typical neuron. Reducing extracellular pH causes a decrease in fluorescence as surface pHluorin-GluR2 is eclipsed. Application of $\mathrm{NH}_{4} \mathrm{Cl}$ rapidly equilibrates cellular pH levels, causing no change in fluorescence at low $\mathrm{pH}$ but a sharp increase at a $\mathrm{pH}$ of 7.4 as all the pHluorin-GluR2 fluorescence in the cell is revealed. e, Hippocampal cultures expressing pHluorin-GluR2 (green) were stained with FM4-64 (red) to visualize actively releasing presynaptic terminals. A magnified area of the dendrite indicated by the dashed box in the top image is shown in the bottom image. Most pHluorin-GluR2 fluorescent puncta are associated with presynaptic terminals $(n=4 ; 73 \pm 3 \%)$. Scale bar, $2 \mu \mathrm{m}$. $f$, Triple labeling of synaptophysin (red), endogenous GluR1 (blue), and pHluorin-GluR2 (green) revealed that pHluorin-GluR2 is targeted to endogenous GluR1-containing synapses (white arrows in overlay image) in transfected neurons $(n=5)$. Scale bar, $10 \mu \mathrm{m}$.

tion of AMPARs in synaptic and extrasynaptic regions of dendritic membrane (Fig. 2a). To ensure that the fluorescence was attributable to surface pHluorin-GluR2, a brief acid wash was included at the start of each experiment. After return to normal pH buffer, baseline images were collected every $20-30$ sec before, during, and for $10 \mathrm{~min}$ after activation of NMDARs.

During NMDA application, we observed a marked difference in the behavior of synaptic and extrasynaptic pHluorin-GluR2. Fluorescence from punctate, synaptic regions was stable during the period of NMDA application but then, in most cells, slowly declined over the course of several minutes. These findings are consistent with the suggestion that AMPARs are slowly removed from synapses after NMDA exposure (Beattie et al., 2000; Ehlers, 2000) (Fig. 2b,c, red tones) and provide a correlate of LTD. In contrast, extrasynaptic pHluorin-GluR2 fluorescence decreased sharply as soon as NMDA was applied. The fluorescence loss during NMDA stimulation suggests a rapid internalization of AMPARs from the extrasynaptic membrane into an acidic intracellular compartment. After NMDA washout, the fluorescence immediately began to recover toward prestimulation levels and then 

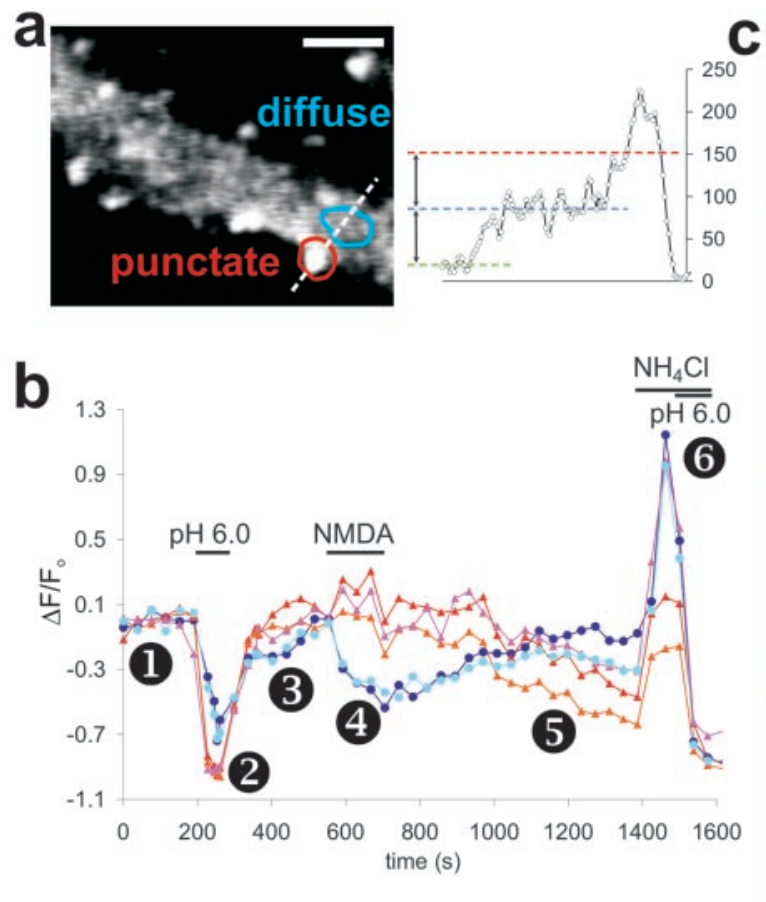

d

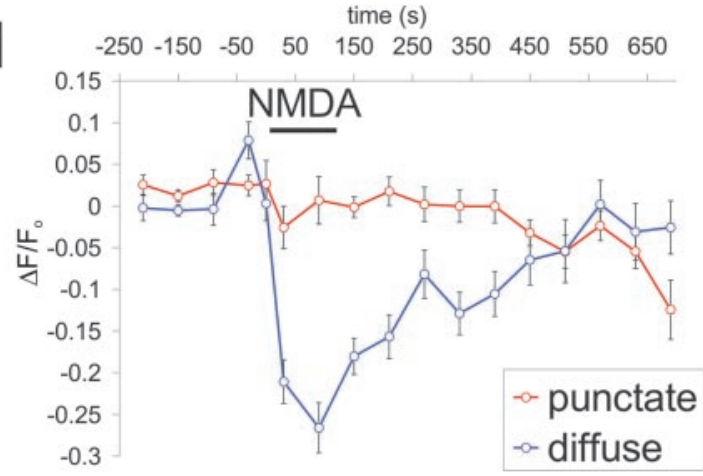

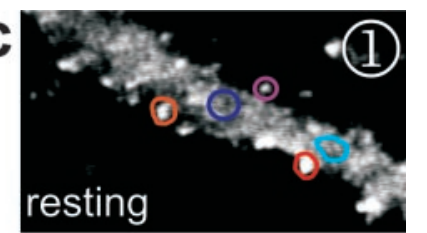
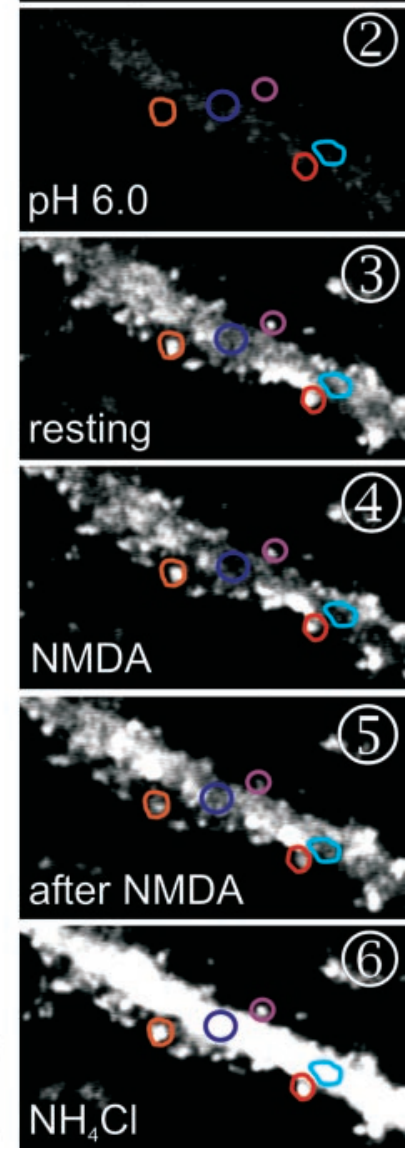

Figure 2. Rapid endocytosis of extrasynaptic AMPARs induced by activation of NMDARs. $a$, Enlarged image of dendritic pHluorin-GluR2 fluorescence labeled with typical punctate and diffuse regions. Scale bar, $4 \mu \mathrm{m}$. Regions for analysis were selected on the basis of relative brightness; as shown by the fluorescence profile plot (dashed white line), puncta (red line) had at least twice the fluorescence of diffuse regions (blue line). $b$, Fluorescence changes at individual dendritic regions shown in images in $c$. Lines with red tones are from typical punctate regions, and blue lines are from diffuse areas. Application of $50 \mu \mathrm{m}$ NMDA caused a slow decrease in punctate fluorescence but a rapid and transient decrease in diffuse fluorescence. $c$, Images from time points corresponding to numbers on the graph. $d$, Binned and averaged fluorescence values from punctate (red) and diffuse (blue) regions during NMDA stimulation. Error bars show \pm SEM ( 6 cells, 28 punctate and 27 diffuse regions).

endocytosis of AMPARs among the plethora of other receptors and subsynaptic proteins present. We have developed a novel approach in which the $\mathrm{pH}$-sensitive GFP variant, ecliptic pHluorin, is tagged to GluR2 to allow us to dynamically analyze trafficking of AMPARs at individual synapses with a much higher spatial and temporal resolution than has previously been possible. By specifically imaging AMPARs at the cell surface during NMDAR activation, we have shown that the endocytosis of extrasynaptic and synaptic AMPARs is subject to differential regulation, as has been suggested for NMDARs ( $\mathrm{Li}$ et al., 2002). The brief pulse of NMDA that we used to evoke AMPAR internalization correlates with Chem-LTD (Lee et al., 1998; Ehlers, 2000), although it should be noted that both synaptic and extrasynaptic NMDARs are activated. Our data indicate that extrasynaptic AMPARs are rapidly endocytosed after NMDA application. Interestingly, pHluorin-GluR2 fluorescence in synaptic regions did not decrease during NMDAR activation, demonstrating that synaptic AMPARs are initially protected from endocytosis. This suggests that the removal of AMPARs from synapses, as occurs during LTD, is preceded by the internalization of AMPARs from extrasynaptic sites. Indeed, a brief increase in extrasynaptic AMPAR endocytosis, as demonstrated here, along with regulated lateral diffusion of synaptic AMPARs out of the PSD (Tardin et al., 2003), could account for a sustained decrease in synaptic strength.

\section{References}

Banker G, Goslin K, eds (1998) Culturing nerve cells, Ed 2. Cambridge, MA: MIT.

Beattie EC, Carroll RC, Yu X, Morishita W, Yasuda H, von Zastrow M, Malenka RC (2000) Regulation of AMPA receptor endocytosis by a signaling mechanism shared with LTD. Nat Neurosci 3:1291-1300.

Blanpied TA, Scott DB, Ehlers MD (2002) Dynamics and regulation of clathrin coats at spe-

restabilized (Fig. 2b,c, blue tones). This subsequent recovery after removal of the stimulus is caused by the movement of pHluorinGluR2 into extrasynaptic regions. The recovery could be attributable to rapid recycling of endocytosed pHluorin-GluR2 or lateral diffusion of pHluorin-GluR2 from adjacent regions of plasma membrane. Both processes have been reported previously (Ehlers, 2000; Borgdorff and Choquet, 2002; Choquet and Triller, 2003). The difference between the dynamics of pHluorin-GluR2 at synaptic and extrasynaptic sites after NMDAR activation is summarized in Figure $2 d$. NMDA had no effect on intracellular $\mathrm{pH}(n=4)$ or on cellular autofluorescence ( $n=3$; data not shown). Therefore, the changes in fluorescence accurately track the internalization of AMPARs.

\section{Discussion}

Synaptic AMPARs are localized within a condensed mass of proteins at the PSD, and it is difficult to conceive of mechanisms that would allow the highly specific, subunit-selective exocytosis and cialized endocytic zones of dendrites and spines. Neuron 36:435-449.

Borgdorff AJ, Choquet D (2002) Regulation of AMPA receptor lateral movements. Nature 417:649-653.

Carroll RC, Beattie EC, von Zastrow M, Malenka RC (2001) Role of AMPA receptor endocytosis in synaptic plasticity. Nat Rev Neurosci 2:315-324.

Choquet D, Triller A (2003) The role of receptor diffusion in the organization of the postsynaptic membrane. Nat Rev Neurosci 4:251-265.

Demaurex N (2002) pH Homeostasis of cellular organelles. News Physiol Sci 17:1-5.

Ehlers MD (2000) Reinsertion or degradation of AMPA receptors determined by activity-dependent endocytic sorting. Neuron 28:511-525.

Harding TC, Geddes BJ, Noel JD, Murphy D, Uney JB (1997) Tetracyclineregulated transgene expression in hippocampal neurones following transfection with adenoviral vectors. J Neurochem 69:2620-2622.

Lee HK, Kameyama K, Huganir RL, Bear MF (1998) NMDA induces longterm synaptic depression and dephosphorylation of the GluR1 subunit of AMPA receptors in hippocampus. Neuron 21:1151-1162.

Lee SH, Liu L, Wang YT, Sheng M (2002) Clathrin adaptor AP2 and NSF 
interact with overlapping sites of GluR2 and play distinct roles in AMPA receptor trafficking and hippocampal LTD. Neuron 36:661-674.

Li B, Chen N, Luo T, Otsu Y, Murphy TH, Raymond LA (2002) Differential regulation of synaptic and extra-synaptic NMDA receptors. Nat Neurosci 5:833-834.

Lissin DV, Gomperts SN, Carroll RC, Christine CW, Kalman D, Kitamura M, Hardy S, Nicoll RA, Malenka RC, von Zastrow M (1998) Activity differentially regulates the surface expression of synaptic AMPA and NMDA glutamate receptors. Proc Natl Acad Sci USA 95:7097-7102.

Malinow R, Malenka RC (2002) AMPA receptor trafficking and synaptic plasticity. Annu Rev Neurosci 25:103-126.

Malinow R, Mainen ZF, Hayashi Y (2000) LTP mechanisms: from silence to four-lane traffic. Curr Opin Neurobiol 10:352-357.

Miesenbock G, De Angelis DA, Rothman JE (1998) Visualizing secretion and synaptic transmission with $\mathrm{pH}$-sensitive green fluorescent proteins. Nature 394:192-195.
Morales M, Goda Y (1999) Nomadic AMPA receptors and LTP. Neuron 23:431-434.

Passafaro M, Piech V, Sheng M (2001) Subunit-specific temporal and spatial patterns of AMPA receptor exocytosis in hippocampal neurons. Nat Neurosci 4:917-926.

Sankaranarayanan S, De Angelis D, Rothman JE, Ryan TA (2000) The use of pHluorins for optical measurements of presynaptic activity. Biophys J 79:2199-2208.

Sheng M, Lee SH (2001) AMPA receptor trafficking and the control of synaptic transmission. Cell 105:825-828.

Shi S, Hayashi Y, Esteban JA, Malinow R (2001) Subunit-specific rules governing AMPA receptor trafficking to synapses in hippocampal pyramidal neurons. Cell 105:331-343.

Tardin C, Cognet L, Bats C, Lounis B, Choquet D (2003) Direct imaging of lateral movements of AMPA receptors inside synapses. EMBO J 22:46564665 . 